\section{OC-019 CLASSICAL AND ALTERNATIVE PATHWAY NUCLEAR FACTOR-KB SIGNALLING DIFFERENTIALLY REGULATE GASTRIC EPITHELIAL RESPONSES TO HELICOBACTER FELIS INFECTION}

doi:10.1136/gut.2011.239301.19

M D Burkitt, 1,* A Varro, ${ }^{2}$ J H Caamano, ${ }^{3}$ D M Pritchard ${ }^{1}$ Department of Gastroenterology, The University of Liverpool, Liverpool, UK; ${ }^{2}$ Department of Cellular and Molecular Physiology, The University of Liverpool, Liverpool, UK; ${ }^{3}$ Unit of Immune Regulation, The University of Birmingham, Birmingham, UK

Introduction Classical pathway NF- $\mathrm{\kappa B}$ signalling is implicated in the pathogenesis of several inflammation associated cancers, including colitis associated colon cancer and Helicobacter associated gastric cancer. However, the role of individual NF- $\mathrm{KB}$ family members and the function of alternative pathway NF- $\kappa B$ signalling have not previously been assessed in this context. We have therefore investigated whether abrogation of classical and alternative pathway NF- $\kappa \mathrm{B}$ signalling altered murine responses to Helicobacter felis infection.

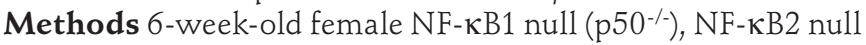
(p52--), c-Rel null and C57BL/6 mice were infected with $H$. felis by oral gavage and culled 6 weeks later. Tissues were processed for histological analysis and immunohistochemistry.

Results $H$. felis infection of wild-type mice resulted in gastric atrophy $29 \%$ fewer parietal cells were observed in infected than in control mice ( $p<0.05,1$-way ANOVA and Holm Sidak post hoc test)), a 1.5 -fold increase in the number of Ki67 positive proliferating cells and no significant change in the number of active caspase 3 positive apoptotic cells. Animals with abrogated classical pathway NF-KB signalling also developed gastric atrophy after $H$. felis infection. However, whereas infected c-Rel null animals showed similar parietal cell, proliferation and apoptotic indices to infected wild-type mice, $\mathrm{p} 50^{-/-}$animals developed more severe pathology with significantly increased inflammation scores ( $p<0.05$, Mann-Whitney $\mathrm{U}$ ) and a more marked $62 \%$ reduction in parietal cell number $(p<0.05$, 1-way ANOVA). This was associated with significant 2.1-fold and 7.6fold increases in the number of proliferating and apoptotic cells, respectively ( $p<0.05,2$-way ANOVA). By contrast, infected p52 $\%$ mice showed much lower inflammation scores than wildtype mice ( $p<0.05, \mathrm{MWU}$ ) following $H$. felis infection and did not develop gastric atrophy, with only $3 \%$ parietal cell loss ( $p<$ 0.05, 1-way ANOVA). In addition, these mice showed no significant changes in proliferation or apoptosis following infection with $H$. felis, and demonstrated similar proliferation and apoptotic indices to untreated wild-type mice.

Conclusion NF- $\mathrm{KB} 1$ mediated signalling protects the gastric mucosa from Helicobacter induced atrophy, whereas alternative pathway NF- $\mathrm{\kappa B}$ signalling is required for the development of both inflammation and atrophy following infection with this organism. This supports the hypothesis that classical and alternative pathway signalling differentially affect long-term outcomes, including carcinogenesis, following $H$. felis infection in C57BL/6 mice.

Competing interests None.

Keywords gastric atrophy, H. pylori, NF-kB. 